\title{
Effects of Psychiatric Factors on Patient-Reported Outcomes after Surgical Correction of Lumbar Degenerative Kyphosis
}

\author{
Tae Sik Goh ${ }^{1}$, Jong Ki Shin ${ }^{1}$, Myung Soo Youn ${ }^{2}$, Jung Sub Lee ${ }^{1}$ \\ ${ }^{1}$ Department of Orthopaedic Surgery, Biomedical Research Institute, Pusan National University School of Medicine, Busan, Korea \\ ${ }^{2}$ Department of Orthopaedic Surgery, Myungeun Hospital, Busan, Korea
}

Study Design: A prospective study.

Purpose: To identify associations between psychiatric factors and patient-reported outcomes after corrective surgery in patients with lumbar degenerative kyphosis (LDK).

Overview of Literature: Thus far, to the best of our knowledge, patient factors that may help predict patient-reported outcomes after corrective surgery for LDK have not been studied.

Methods: We prospectively investigated 46 patients with LDK who underwent surgical correction with a minimum follow-up of 2 years. Demographic data were collected. Short form-36, mental component scores (MCS), physical component scores (PCS), Scoliosis Research Society-22 (SRS-22) scores, and Roland-Morris Disability Questionnaire (RMDQ) scores were determined before the surgery and after 2 years of follow-up. Psychiatric conditions were preoperatively evaluated using the Zung depression scale (ZDS) and Zung anxiety scale (ZAS). Patients were divided into two groups (with or without psychiatric issues), according to baseline ZDS and ZAS scores.

Results: Patients included 43 women and 3 men. Twelve patients were deemed to have psychiatric problems (P group) and 34 patients had no psychiatric problems (NP group). No significant intergroup differences were found in MCS, PCS, SRS-22, and RMD0 scores preoperatively. However, at the 2-year follow-up, a significant intergroup difference was observed between PCS and RMDO scores. Multiple regression analysis revealed that only the presence of a preoperative psychiatric problem can predict PCS and RMDO scores. Other factors, such as, gender, age, body mass index, bone mineral density, osteotomy site, number of fusion segments, and instrumented levels did not affect PCS or RMDQ scores.

Conclusions: The presence of a psychiatric factor may be an important risk factor underlying poor physical and pain scores after corrective surgery in patients with LDK. The findings presented here suggest that psychiatric factors should be evaluated prior to surgery for determining the risk of a poor outcome.

Keywords: Lumbar vertebrae; Degenerative disease; Kyphosis; Outcome; Psychiatric diagnosis

Received Mar 20, 2017; Revised Apr 19, 2017; Accepted May 11, 2017

Corresponding author: Jung Sub Lee

Department of Orthopaedic Surgery, Medical Research Institute, Pusan National University Hospital, Pusan National University School of Medicine, 179 Gudeok-ro, Seo-gu, Busan 49241, Korea

Tel: +82-51-240-7248, Fax: +82-51-247-8395, E-mail: jungsublee@pusan.ac.kr 


\section{Introduction}

Lumbar degenerative kyphosis (LDK) is a characteristic disease featuring dynamic stooping. Loss of lordosis or kyphosis in the lumbar spine results in sagittal imbalance, which leads to muscle fatigue and intractable back pain, particularly while standing and walking [1-4]. In recent years, demands for active treatments aimed at improving the quality of life of patients with LDK have increased because of the increased number of elderly patients. Factors known to affect clinical outcomes after the surgical correction of long-level spinal deformity include incomplete correction, fixation failure, and nonunion. However, despite the presence of the correction loss, in some cases, good clinical outcomes are achieved. Furthermore, some patients with LDK who achieve sagittal balance after surgical correction continue to suffer from intractable back pain, which suggests that the degree of satisfaction with the surgical outcome is not directly associated with achieving sagittal balance and that other factors may contribute to clinical outcomes. However, it may be difficult to determine predictive outcome factors in patients with LDK treated by an extensive surgery such as pedicle subtraction osteotomy (PSO).

We considered a possible contribution of psychiatric factors, which have been extensively studied in spinal diseases [5-10]. Several authors reported that depression and other abnormal psychological conditions are inadequate predicting poor outcomes after spinal surgery or rehabilitation. However, little information is available on effects of preoperative psychiatric factors on outcomes after corrective surgery in patients with LDK. Therefore, the purpose of this study was to evaluate the association between psychiatric factors and postoperative outcomes after corrective surgery in patients with LDK.

\section{Materials and Methods}

This study and all procedures were approved by the Institutional Review Board of Pusan National University Hospital (PNUH-IRB no., 2009053).

We prospectively studied 52 patients with LDK treated by PSO at two spine centers from January 2009 to December 2014. Six patients were lost to follow-up and thus excluded; therefore, 46 patients with a minimum followup of 2 years were enrolled in this study, approved by our institutional Clinical Research Ethics Committee. Patients with a history of spinal surgery, coxofemoral pathology, or post-traumatic kyphosis were excluded.

Operative treatment was performed on patients who complained of intractable low back pain with kyphotic deformity and subjectively intolerable difficulties in standing independently, walking, or during the activities required for daily living. PSO with or without posterior lumbar interbody fusion (PLIF) was performed in all patients. PLIF was performed for patients who had symptomatic spinal stenosis requiring decompression. No patients underwent anterior lumbar interbody fusion. Postoperatively, patients wore a thoraco-lumbo-sacral-orthosis brace for 3 months and were allowed to perform daily activities, but not to perform any type of work.

Clinical outcomes were evaluated using short form-36 [11], mental component scores (MCS), physical component scores (PCS), Scoliosis Research Society-22 (SRS22) [12], and the Roland-Morris Disability Questionnaire (RMDQ) [13] preoperatively and at 2 years postoperatively. At the 2-year follow-up, all patients were contacted by telephone and asked to complete a questionnaire.

Psychiatric conditions were preoperatively evaluated using the Zung depression scale (ZDS) [14] and the Zung anxiety scale (ZAS) [15]. ZDS scores range from 20 "no depression" to 80 "major depression," and a cut-off value of $>49$ indicates the presence of significant depressive symptoms. ZAS scores also range from 20 "no anxiety" to 80 "extreme anxiety," and a cut-off value of $>44$ indicates significant anxious symptoms. Patients were divided into two different cohorts (with or without psychiatric problems), according to their baseline ZDS and ZAS scores. In this study, patients with no psychiatric problems were considered as those with a ZDS score of $<50$ and/or a ZAS score of $<45$.

Possible risk factors affecting outcomes evaluated were age, sex, body mass index (BMI), PSO sites, number of fusion segments, upper instrumented vertebra (UIV), lower instrumented vertebra (LIV), lumbar spine bone mineral density (BMD), and femoral neck BMD.

Statistical analyses were performed using SPSS ver. 11.5 for Windows (SPSS Inc., Chicago, IL, USA). Results are expressed as mean values \pm standard deviations. Regression analysis was performed for identifying predictors of outcome scores. Initially, univariate regression was used to test for associations between variables, and then forward stepwise multiple regression analysis was used for developing a prediction model. The significances of differences 
Table 1. Details of the patients

\begin{tabular}{|c|c|c|c|}
\hline Characteristic & $P$ group $^{\text {a) }}(n=12)$ & NP group ${ }^{\text {b) }}(n=34)$ & $p$-value \\
\hline Age (yr) & $60.8 \pm 6.4$ & $60.9 \pm 6.1$ & 0.961 \\
\hline Sex & & & 0.701 \\
\hline Female & 11 & 32 & \\
\hline Male & 1 & 2 & \\
\hline Body mass index $\left(\mathrm{kg} / \mathrm{m}^{2}\right)$ & $24.5 \pm 3.2$ & $23.8 \pm 2.3$ & 0.406 \\
\hline Pedicle subtraction osteotomy site L1/2/3/4 & $3 / 6 / 2 / 1$ & $4 / 21 / 6 / 3$ & 0.744 \\
\hline Fusion segments (no.) & $7.4 \pm 1.4$ & $7.1 \pm 1.3$ & 0.424 \\
\hline Lower instrumented vertebra $\mathrm{L} 3 / 4 / 5 / \mathrm{S} / \mathrm{I}$ & $0 / 3 / 3 / 5 / 1$ & $1 / 8 / 11 / 12 / 2$ & 0.952 \\
\hline Lumbar spine BMD $\left(\mathrm{g} / \mathrm{cm}^{2}\right)$ & $0.751 \pm 0.046$ & $0.750 \pm 0.051$ & 0.901 \\
\hline Femoral neck BMD $\left(\mathrm{g} / \mathrm{cm}^{2}\right)$ & $0.734 \pm 0.044$ & $0.738 \pm 0.048$ & 0.816 \\
\hline Preoperative RMD0 & $20.8 \pm 2.2$ & $19.9 \pm 1.8$ & 0.208 \\
\hline Preoperative SRS-22 & $40.5 \pm 9.1$ & $39.9 \pm 5.4$ & 0.779 \\
\hline Preoperative PCS & $28.6 \pm 6.5$ & $30.5 \pm 5.8$ & 0.353 \\
\hline Preoperative MCS & $37.7 \pm 7.5$ & $38.1 \pm 5.4$ & 0.824 \\
\hline Two-year follow-up RMD0 & $18.9 \pm 2.7$ & $15.2 \pm 2.2$ & $<0.001$ \\
\hline Two-year follow-up SRS-22 & $61.2 \pm 8.6$ & $63.1 \pm 7.6$ & 0.465 \\
\hline Two-year follow-up PCS & $42.0 \pm 6.7$ & $49.1 \pm 6.0$ & 0.001 \\
\hline Two-year follow-up MCS & $49.1 \pm 5.0$ & $51.5 \pm 6.3$ & 0.235 \\
\hline Improvement RMDO & $1.8 \pm 1.5$ & $4.7 \pm 2.4$ & 0.001 \\
\hline Improvement SRS-22 & $20.7 \pm 8.9$ & $23.2 \pm 8.1$ & 0.363 \\
\hline Improvement PCS & $13.4 \pm 5.9$ & $18.6 \pm 7.5$ & 0.035 \\
\hline Improvement MCS & $11.4 \pm 6.6$ & $13.4 \pm 7.9$ & 0.437 \\
\hline
\end{tabular}

Values are presented as mean \pm standard deviation or number.

L, lumbar; S, sacral; I, iliac; BMD, bone mineral density; RMD0, Roland-Morris Disability Questionnaire; SRS-22, Scoliosis Research Society-22; PCS, physical component score; MCS, mental component score.

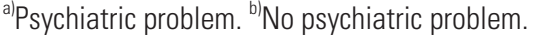

between P group (with a psychiatric problem) and NP groups (without a psychiatric problem) were assessed using the $t$-test or $\chi^{2}$ test. All $p$-values $<0.05$ were considered to be statistically significant.

\section{Results}

The patients included 43 women and 3 men with the overall mean age of $61.1 \pm 7.8$ years. Overall mean preoperative MCS, PCS, SRS-22, and RMDQ scores improved from 38.0, 30.0, 40.0, and 20.2 to 50.9, 47.2, 62.6, and 16.2, respectively, at the 2-year follow-up. Overall mean preoperative ZDS and ZAS scores were 34.1 and 33.7, respectively.

On the basis of their baseline ZDS and ZAS scores, 12 patients (26.1\%) were deemed to have a psychiatric prob-
Table 2. Multiple regression analysis in lumbar degenerative kyphosis patients

\begin{tabular}{lccc} 
Variable & Coefficient & $t$-value & $p$-value \\
Two-year follow-up RMD0 & & & \\
Psychiatric factor & 3.711 & 4.708 & $<0.001$ \\
\hline Constant & 15.206 & & \\
Two-year follow-up PCS & & & \\
\hline Psychiatric factor & -7.088 & -3.403 & 0.001 \\
\hline Constant & 49.088 & & \\
\hline
\end{tabular}

RMDQ, Roland-Morris Disability Questionnaire; PCS, physical component score.

lem (P group) and 34 patients (73.9\%) were deemed to have no psychiatric problems (NP group). No significant differences were observed in MCS, PCS, SRS-22, and 
RMDQ preoperative scores between the groups (Table 1).

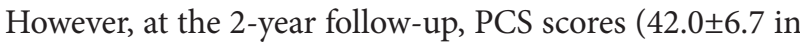
the $\mathrm{P}$ group and $49.1 \pm 6.0$ in the NP group) and RMDQ scores $(18.9 \pm 2.7$ in the P group and $15.2 \pm 2.2$ in the NP group) were shown to significantly differ.

Multiple regression analysis results demonstrated that only the presence of a preoperative psychiatric problem independently predicted PCS and RMDQ scores (Table 2). Other factors, such as gender, age, BMI, BMD, osteotomy site, number of fusion segments, UIV, and LIV were not found to affect PCS or RMDQ scores.

\section{Discussion}

LDK is one of the most common spinal deformities encountered in Asia [16]. Surgical procedures for the treatment of LDK include posterior instrumentation, combined anterior and posterior surgery, and various types of corrective osteotomies including PSO [17-19]. However, despite the application of these procedures, not all patients experience pain reduction, a better quality of life, or disability reduction postoperatively $[1,2,4,20]$, which has placed emphasis on optimizing factors that may maximize the benefits of surgery. Traditionally, the optimization has been limited to conservative therapy trials, such as activity modification, analgesics, spinal manipulation, injections, and physical therapy, prior to pursuing surgical options. Nevertheless, patients with intractable low back pain, kyphotic deformity, and/or dynamic stooping and difficulties in performing activities required for daily living often subsequently undergo surgical interventions [4], and a subset of these patients experience no improvement postoperatively. This has led several authors to propose that intrinsic patient characteristics, such as depression and anxiety, might play a role in the failure to achieve a meaningful improvement postoperatively.

Patients with psychological comorbidities are known to suffer from a long-term disability due to low back pain $[7,21]$, and several authors have suggested that patients with a psychological comorbidity, such as depression or anxiety, experience poorer outcomes after different spinal procedures [22,23]. Arpino et al. [24] assessed the effects of depression on 1-year pain scores and found that patients with fewer depressive symptoms achieved a significantly better improvement in pain symptoms. Similarly, Schade et al. [25] found that patients with fewer depressive symptoms or occupational stress are more likely to return to work within 2 years following laminectomy. However, despite these reports, effects of psychiatric predispositions on validated disability and quality of life outcome measures postoperatively remains unstudied.

In this study, although the presence of a psychiatric factor was not shown to negatively affect the postoperative MCS or general health (SRS-22) scores, patients with LDK with a psychiatric problem were found to be less likely to achieve clinically significant improvement in terms of physical disability and pain scores (e.g., PCS and RMDQ) following corrective osteotomy. Moreover, multiple regression analysis showed that only the presence of a psychiatric factor can significantly predict PCS and RMDQ scores. We do not know how the presence of a psychiatric factor predisposes patients to pain, and it is possible that these patients tend to over-report pain. Nevertheless, our findings indicate that patients with a psychiatric factor should be counseled prior to surgery on risks of physical disability and poor outcomes regarding pain.

Several limitations of our study should be noted. First, because the aim of this study was to investigate preoperative psychiatric factors affecting postoperative outcomes in patients with LDK treated by PSO, we did not measure radiological changes, such as sagittal spinal balance or degree of correction angle. Moreover, we did not define criteria for UIV, site of PSO, or fusion level. However, impacts of these factors on the surgical treatment of sagittal deformity have not been clearly defined. Furthermore, the number of patients enrolled was too small to reach conclusions regarding the effects of the psychiatric status on clinical outcome scores. Accordingly, a further larger study is needed to confirm our results. Third, we did not assess psychiatric disorder-related medical histories, and preoperative antidepressive medication or psychiatric behavior treatment could have improved postoperative outcomes. Fourth, data recovered were subjective because 2 -year follow-ups were conducted by telephone, and no objective data that might be correlated with perceived symptoms were obtained. Future prospective studies incorporating objective measures, such as radiological findings, may provide more insights.

This study may help in clarifying effects of psychiatric problems on patient outcomes after corrective osteotomy for LDK. Well-accepted numeric scales were used for determining depression and anxiety levels, and the effects of depression and anxiety on validated outcome measures of the quality of life (PCS, MCS, and SRS-22) and 
pain (RMDQ) were assessed. Therefore, we hope that the findings of the present study will aid in the optimization of clinical outcomes for surgically-treated patients with LDK.

\section{Conclusions}

We showed here that the presence of a psychiatric problem before corrective osteotomy is associated with poorer physical and pain scores in patients with LDK. Furthermore, our findings suggest that patients with a psychiatric factor should be counseled preoperatively regarding the risk of physical disability and of poor outcome with respect to pain.

\section{Conflict of Interest}

No potential conflict of interest relevant to this article was reported.

\section{Acknowledgments}

This work was supported for two years by Pusan National University Research Grant.

\section{References}

1. Chang DG, Ha KY, Kim YH, Lee EW. Spinopelvic alignment by different surgical methods in the treatment of degenerative sagittal imbalance of the lumbar spine. Clin Spine Surg 2017;30:E390-7.

2. Kim WJ, Kang JW, Kang SI, et al. Factors affecting clinical results after corrective osteotomy for lumbar degenerative kyphosis. Asian Spine J 2010;4:7-14.

3. Lee JH, Kim KT, Suk KS, et al. Analysis of spinopelvic parameters in lumbar degenerative kyphosis: correlation with spinal stenosis and spondylolisthesis. Spine (Phila Pa 1976) 2010;35:E1386-91.

4. Lee SH, Kim KT, Suk KS, Lee JH, Seo EM, Huh DS. Sagittal decompensation after corrective osteotomy for lumbar degenerative kyphosis: classification and risk factors. Spine (Phila Pa 1976) 2011;36:E538-44.

5. Aalto TJ, Malmivaara A, Kovacs F, et al. Preoperative predictors for postoperative clinical outcome in lumbar spinal stenosis: systematic review. Spine (Phila Pa 1976) 2006;31:E648-63.

6. Celestin J, Edwards RR, Jamison RN. Pretreatment psychosocial variables as predictors of outcomes following lumbar surgery and spinal cord stimulation: a systematic review and literature synthesis. Pain Med 2009;10:639-53.

7. Chaichana KL, Mukherjee D, Adogwa O, Cheng JS, McGirt MJ. Correlation of preoperative depression and somatic perception scales with postoperative disability and quality of life after lumbar discectomy. J Neurosurg Spine 2011;14:261-7.

8. Kang SS, Lee JS, Shin JK, Lee JM, Youn BH. The association between psychiatric factors and the development of chronic dysphagia after anterior cervical spine surgery. Eur Spine J 2014;23:1694-8.

9. Kim TW, Oh CH, Shim YS, et al. Psychopathological influence of lumbar disc herniation in male adolescent. Yonsei Med J 2013;54:813-8.

10. Sinikallio S, Lehto SM, Aalto T, Airaksinen O, Kroger H, Viinamaki H. Depressive symptoms during rehabilitation period predict poor outcome of lumbar spinal stenosis surgery: a two-year perspective. BMC Musculoskelet Disord 2010;11:152.

11. Han CW, Lee EJ, Iwaya T, Kataoka H, Kohzuki M. Development of the Korean version of ShortForm 36-Item Health Survey: health related QOL of healthy elderly people and elderly patients in Korea. Tohoku J Exp Med 2004;203:189-94.

12. Lee JS, Lee DH, Suh KT, Kim JI, Lim JM, Goh TS. Validation of the Korean version of the Scoliosis Research Society: 22 questionnaire. Eur Spine J 2011;20:1751-6.

13. Lee JS, Lee DH, Suh KT, Kim JI, Lim JM, Goh TS. Validation of the Korean version of the RolandMorris Disability Questionnaire. Eur Spine J 2011;20:2115-9.

14. Zung WW. A self-rating depression scale. Arch Gen Psychiatry 1965;12:63-70.

15. Zung WW. A rating instrument for anxiety disorders. Psychosomatics 1971;12:371-9.

16. Takemitsu Y, Harada Y, Iwahara T, Miyamoto M, Miyatake Y. Lumbar degenerative kyphosis: clinical, radiological and epidemiological studies. Spine (Phila Pa 1976) 1988;13:1317-26.

17. Berjano P, Aebi M. Pedicle subtraction osteotomies (PSO) in the lumbar spine for sagittal deformities. Eur Spine J 2015;24 Suppl 1:S49-57.

18. Le Huec JC, Aunoble S. Pedicle subtraction osteotomy for sagittal imbalance. Eur Spine J 2012;21:1896- 
7.

19. Roussouly P, Nnadi C. Sagittal plane deformity: an overview of interpretation and management. Eur Spine J 2010;19:1824-36.

20. Jang JS, Lee SH, Kim JM, Min JH, Han KM, Maeng DH. Can patients with sagittally well-compensated lumbar degenerative kyphosis benefit from surgical treatment for intractable back pain? Neurosurgery 2009;64:115-21.

21. Waddell G, Main CJ, Morris EW, Di Paola M, Gray IC. Chronic low-back pain, psychologic distress, and illness behavior. Spine (Phila Pa 1976) 1984;9:209-13.
22. Turner RS, Leiding WC. Correlation of the MMPI with lumbosacral spine fusion results: prospective study. Spine (Phila Pa 1976) 1985;10:932-6.

23. Uomoto JM, Turner JA, Herron LD. Use of the MMPI and MCMI in predicting outcome of lumbar laminectomy. J Clin Psychol 1988;44:191-7.

24. Arpino L, Iavarone A, Parlato C, Moraci A. Prognostic role of depression after lumbar disc surgery. Neurol Sci 2004;25:145-7.

25. Schade V, Semmer N, Main CJ, Hora J, Boos N. The impact of clinical, morphological, psychosocial and work-related factors on the outcome of lumbar discectomy. Pain 1999;80:239-49. 